\title{
Review
}

\section{Should the Augmentation Index be Normalized to Heart Rate?}

\author{
Lee Stoner $^{1}$, James Faulkner ${ }^{1}$, Andrew Lowe ${ }^{2}$, Danielle M. Lambrick ${ }^{3}$, Joanna M. Young ${ }^{4}$, Richard Love ${ }^{3}$ \\ and David S. Rowlands ${ }^{1}$ \\ ${ }^{1}$ Massey University, School of Sport and Exercise, Wellington, New Zealand \\ ${ }^{2}$ Auckland University of Technology, Institute for Biomedical Technologies, New Zealand \\ ${ }^{3}$ Massey University, Institute of Food Nutrition and Human Health, Palmerston North, New Zealand \\ ${ }^{4}$ Lipid and Diabetes Research Group, Diabetes Research Institute, Christchurch Hospital Campus, Christchurch, New Zealand
}

\begin{abstract}
Pulse wave analysis (PWA) is widely used to investigate systemic arterial stiffness. The augmentation index (AIx), the primary outcome derived from PWA, is influenced by the mean arterial pressure (MAP), age, gender and heart rate (HR). Gender- and age-specific reference values have been devised, and it is recommended that the MAP be used as a statistical covariate. The AIx is also commonly statistically adjusted to a HR of $75 \mathrm{~b} \cdot \mathrm{min}^{-1}$; however, this approach may be physiologically and statistically inappropriate. First, there appears to be an important physiological chronic interaction between HR and arterial stiffness. Second, the method used to correct to HR assumes that the relationship with AIx is uniform across populations. A more appropriate practice may be to include HR as an independent predictor or covariate; this approach is particularly recommended for longitudinal studies, in which changes in HR may help to explain changes in arterial stiffness.
\end{abstract}

JAtheroscler Thromb, 2014; 21:11-16.

Key words: Pulse wave analysis, Arterial stiffness, Wave reflections, Heart rate, Non-invasive, Cardiovascular disease, Blood pressure, Analysis, Correction

\section{Introduction}

Pulse wave analysis (PWA) has emerged as a noninvasive, valid $^{1)}$, reliable ${ }^{2)}$ and widely used technique for evaluating the augmentation index (AIx), an indicator of systemic arterial stiffness. A recent PubMed database search (June 07, 2013) revealed 1,902 articles that utilized the PWA technique. The popularity of the PWA is not surprising considering that the method is inexpensive, portable and simple to conduct. Nevertheless, the most appropriate mathematical expression off AIx is at present unclear. In particular, there is uncertainty regarding the normalization of the AIx to heart rate (AIx@75).

Address for correspondence: Lee Stoner, Massey University, School of Sport and Exercise, Wellington, New Zealand

E-mail: dr.l.stoner@gmail.com

Received: June 18, 2013

Accepted for publication: September 18, 2013

\section{Measurement}

A number of commercial devices are available to automate PWA assessments. Such devices apply tonometry, oscillometry, volume plethysmography or photo plethysmography to measure the pulse waveform, typically at a peripheral site. Applanation tonometry is considered the gold standard and is the most widely used technique ${ }^{3)}$. The most commonly used device, the SphygmoCor (AtCor Medical, Sydney, Australia), utilizes applanation tonometry to record the radial artery pulse waveform. The peak and trough of the radial pulse wave is calibrated to the systolic and diastolic blood pressure measured in the brachial artery, and a generalized transfer factor is then used to generate the corresponding aortic arterial waveform ${ }^{4}$.

\section{Interpretation of the Central Pulse Wave}

Fig. 1 depicts the typical features of the aortic pulse pressure waveform, from which the central 


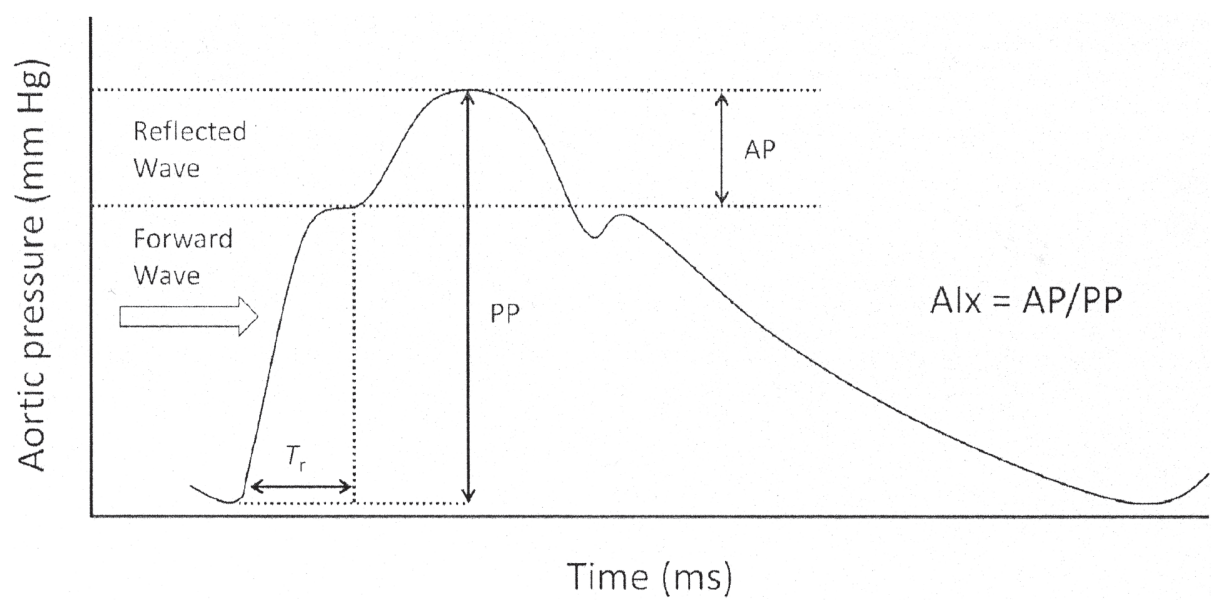

Fig. 1. Aortic pulse pressure waveform.

The systolic and diastolic pressures are the peak and trough of the waveform. The augmentation pressure (AP) is the additional pressure added to the forward wave by the reflected wave. The augmentation index is defined as the $\mathrm{AP}$ as a percentage of the pulse pressure (PP). The time to reflection $(T r)$ is calculated as the time from the onset of the ejected pulse waveform to the onset of the reflected wave.

blood pressure, arrival time of the reflected waves at the central aorta $(T \mathrm{r})$, augmentation pressure (AP) and AIx can be derived. Tr represents the time from the onset of the ejected pulse waveform to the onset of the reflected wave and is a surrogate for aortic pulse wave velocity, i.e., a shorter $\operatorname{Tr}$ indicates increased aortic arterial stiffness ${ }^{5)}$. The AP is normally interpreted as the additional aortic systolic pressure generated by the return of the reflected waves at the central aorta, expressed in absolute terms ${ }^{3)}$. The AIx is the AP as a percentage of the central pulse pressure and is a composite measurement of aortic wave reflection and central arterial stiffness ${ }^{6}$.

\section{Variables Influencing the Alx}

The AIx directly increases with $\mathrm{MAP}^{7)}$ and age ${ }^{8)}$, is higher in women than in men ${ }^{8)}$ and is inversely related to body height ${ }^{9)}$ and heart rate (HR $)^{10,11)}$. For this reason, gender- ${ }^{8)}$ and age-specific ${ }^{8)}$ reference values have been devised. Furthermore, it is recommended that the MAP be used as a covariate if the populations significantly differ ${ }^{12)}$. Statistically adjusting for differences in HR has also been recommended ${ }^{10,11)}$. Based on a correction factor derived from two studies ${ }^{10,11)}$, the SphygmoCor automatically adjusts the AIx at an inverse rate of $4.8 \%$ for each $10 \mathrm{~b} \cdot \mathrm{min}^{-1}$ increment (for HR values between 40 and $110 \mathrm{~b} \cdot \mathrm{min}^{-1}$ ). In a study conducted in 22 subjects (age range: $21-84 \mathrm{y}$ ) with permanent cardiac pacemakers, incremental pacing (from 60 to $110 \mathrm{~b} \cdot \mathrm{min}^{-1}$ ) altered the AIx in a lin- ear $(r=-0.76)$ manner at a rate of $-3.9 \%$ for each 10 $\mathrm{b} \cdot \mathrm{min}^{-1}$ increment ${ }^{11)}$. A separate study by the same group $^{10)}$ reported similar findings in 20 subjects (age range: $20-72$ y) undergoing routine cardiac catheterization. Incremental pacing (via the right atrium at 80,100 and $120 \mathrm{~b} \cdot \mathrm{min}^{-1}$ ) resulted in a linear decline $(r=-0.70)$ in the AIx of $5.6 \%$ for each $10 \mathrm{~b} \cdot \mathrm{min}^{-1}$ increment. The slope was steeper than that observed in the previous study, indicating some variability.

\section{Reliability of the Alx and Influence of Normalization to HR}

Any valid technique utilized to measure physiological variables must be reproducible. The Intraclass Correlation Coefficient (ICC) values for repeated measurements obtained at hourly or weekly intervals

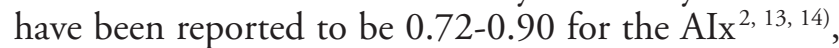
which compares favorably with the values (ICC: $0.92-$ 0.97) reported for the carotid-femoral pulse wave velocity $^{15-17)}$. Unfortunately, few trials have reported the reproducibility of HR-corrected Aix values ${ }^{2,18,19)}$; only one study, as far as we are aware, reported both AIx and AIx@75 2). Papaioannou et al. ${ }^{2)}$ reported that the within-day ICC for the AIx improved from a mean $(\mathrm{SD})$ of $0.86 \pm 0.11$ to $0.90 \pm 0.08$ when normalized to the HR, while the between-day ICC improved from $0.72 \pm 0.19$ to $0.75 \pm 0.15$. These findings, obtained in 22 healthy patients $(36.4 \pm 13.7 \mathrm{y}$; 10 men), must be corroborated. The reliability of the AIx may be further improved by following standard- 
ized guidelines ${ }^{12)}$.

\section{Validity of the Alx and Influence of Normalization to HR}

Arterial stiffness is an important determinant of cardiovascular risk and can be assessed using a number of techniques, including local arterial compliance, pulse wave velocity and PWA. The AIx, which is derived from PWA, indicates the contribution made by the reflected pressure wave to the ascending aortic pressure waveform ${ }^{6}$. The amplitude and speed of the reflected wave are dependent upon arterial stiffness and the AIx thus reflects systemic arterial stiffness ${ }^{6}$. There is evidence that increased aortic wave reflections have adverse effects on ventricular afterload and coronary perfusion, and the pathological role of aortic wave reflections has been demonstrated in several diseases $^{3,20,21)}$. Furthermore, increased central arterial wave reflections have been reported to be significantly correlated to the degree of coronary artery disease ${ }^{22}$ and to independently predict cardiovascular risks and mortality ${ }^{1)}$.

We conducted a PubMed database search (Jan $23,2013)$ to identify interventional or case control studies reporting both AIx and AIx@75 values. Twelve studies were identified and examined to determine whether normalizing AIx to HR influences the interpretation of the findings (Table 1). Eight studies reported significantly improved between-group/condition differences using the AIx@75 versus the AIx alone $^{23-29)}$, two studies reported no significant differences using the AIx but a significant difference using AIx@75 ${ }^{30,31)}$, one study reported a decrease in significance using the AIx@75 ${ }^{32}$ and one study reported a change in the direction of the effect ${ }^{33)}$. Given these findings, careful interpretation of the physiological significance of normalization to HR is warranted.

\section{Is it Physiologically Appropriate to Normalize the Alx to HR?}

Augmentation of the central systolic pressure is influenced by the $\mathrm{HR}$, as an increase in the HR will decrease the absolute duration of systole, effectively shifting the reflected wave into diastole, thereby reducing the $\mathrm{AIx}^{10,11)}$. Wilkinson et al. ${ }^{10)}$ argued that the reduction in AIx associated with cardiac pacing is a mathematical manifestation of a shorter ejection duration and subsequent altered pulse contour, rather than decreased arterial stiffness per se. In effect, this view attributes changes in the AIx to altered cardiac input rather than arterial properties, of which arterial stiff- ness is of primary interest. In support, $T \mathrm{r}$, a measure of the aortic pulse wave velocity, did not change significantly with cardiac pacing ${ }^{10}$. In contrast, an earlier study by the same group ${ }^{11)}$ reported a trend toward a decrease in $T \mathrm{r}$ associated with cardiac pacing from $60-110 \mathrm{~b} \cdot \mathrm{min}^{-1}$ (137 $\mathrm{ms}$ cf. $\left.134 \mathrm{~ms}, p=0.09\right)$. This trend is further supported by the findings of Lantelme et al. ${ }^{34)}$ who reported an increased aortic pulse wave velocity with cardiac pacing from $60 \mathrm{~b} \cdot \mathrm{min}^{-1}$ to 100 $\mathrm{b} \cdot \mathrm{min}^{-1}(13.5 \mathrm{~m} / \mathrm{s}$ cf. $15.1 \mathrm{~m} / \mathrm{s}, p=0.01)$. The latter two studies suggest that increased aortic arterial stiffness, that is the change in AIx, is at least partly attributable to changes in arterial properties rather than cardiac input wave morphology. It should be noted, however, that the reproducibility of $T \mathrm{r}$ has been reported to be fair to good (ICC: $0.43-0.84)^{2,13,14)}$, and, given the low sample size of the two aforementioned studies ( $n=20$ and 22, respectively), these findings must be interpreted with caution.

Stefanadis et al. (1998) reported reduced local aortic stiffness and increased distensibility during incremental ventricular pacing ${ }^{35)}$, which stands in further contrast to the results of similar or increased stiffness reported by Wilkinson ${ }^{10,11)}$ and Lantelme ${ }^{34)}$. Conversely, an observational study $(n=253)$ reported that a high HR is strongly associated with reduced carotid artery distension and an elevated aortic pulse wave velocity, even after adjusting for age and blood pressure $^{36)}$. These discrepant findings imply that the relationship between HR and central arterial stiffness may be altered if the HR remains chronically elevated. Furthermore, these findings suggest that it may be appropriate to normalize to the HR for a given patient if acute serial measurements are obtained and compensation for changes in wave morphology (including ventricular ejection) due to the HR is being attempted. However, normalization of serial measurements obtained in a given patient over a prolonged time course requires careful consideration, as do comparisons between patients.

\section{Is it Statistically Appropriate to Normalize the Alx to HR?}

The following questions must be addressed: (1) Was the correct statistical model used to establish the current linear interpolation normalization factor? (2) Currently, the AIx is corrected to the HR using linear interpolation; is this method appropriate? (3) Is linear interpolation appropriate physiologically? In order to answer these questions, we must first reanalyze the two studies by Wilkinson et al. ${ }^{10,11)}$, from which the correction factor is derived. These two studies regressed 


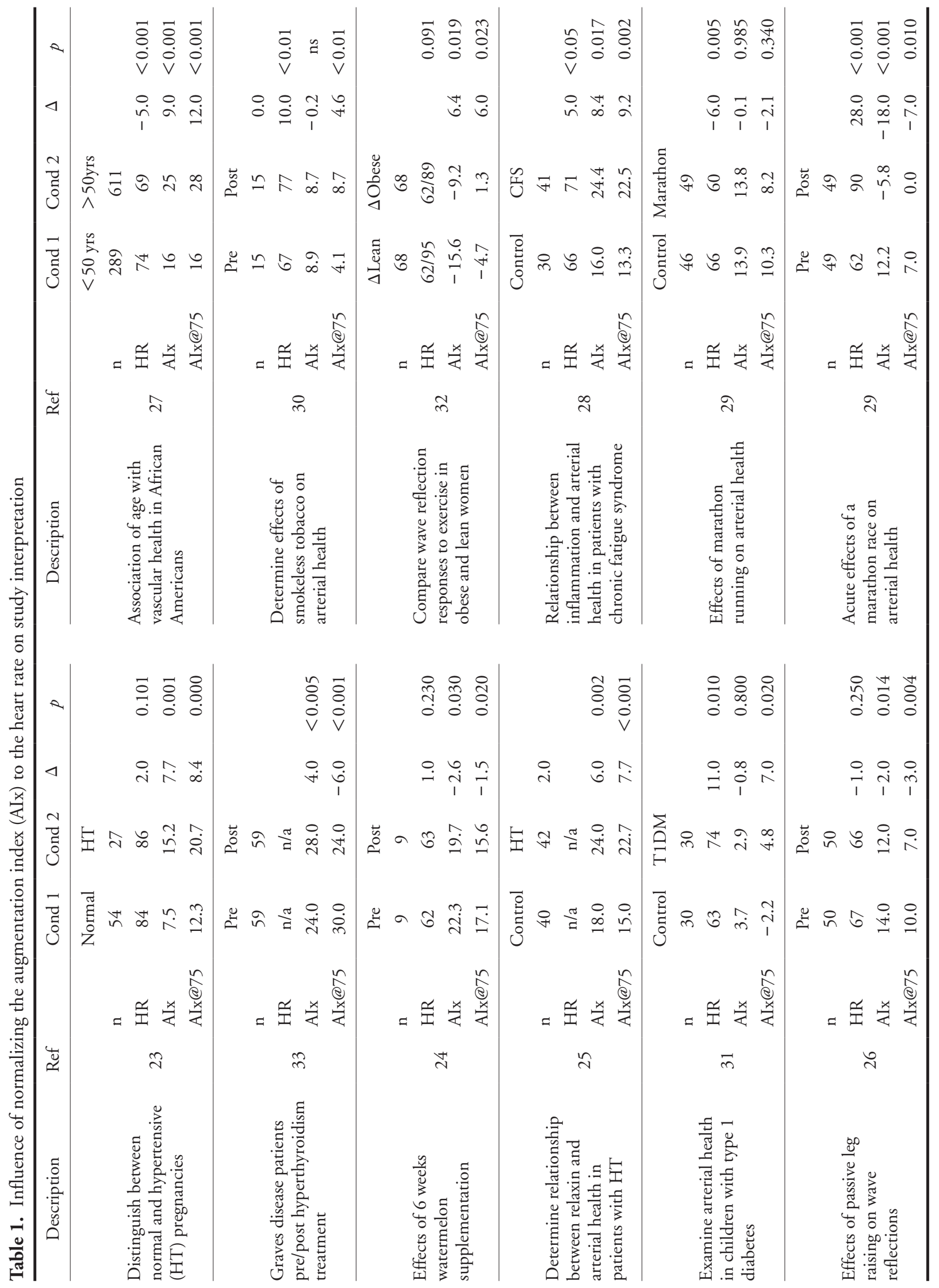


the group mean HR against the group mean AIx using a general linear model (GLM). A basic assumption of GLM is the independence of error, which was violated by Wilkinson et al. via the use of group means. This approach would have removed the between-subjects component of variability and may also have hidden the true nature of the relationship between $X(H R)$ and Y (AIx). A more suitable method would have been hierarchical linear modeling ${ }^{37)}$, which includes a random subject effect to account for correlations among the data. Second, the use of linear interpolation assumes that the relationship between the HR and AIx is the same for all populations, which cannot be ascertained from two small sample studies ${ }^{15,16)}$. Lastly, a cross-sectional study found a positive relationship between HR and arterial stiffness ${ }^{36}$, which is at odds with the negative relationship observed in pacing studies $^{10,11,35)}$. These findings suggest that there may be an important physiological chronic interaction between $\mathrm{HR}$ and arterial stiffness. Therefore, while normalizing to HR using linear interpolation may decrease shortterm measurement variability, this practice may potentially lead to inferential error. A more appropriate practice may be to include HR as an independent predictor or covariate; this approach is particularly recommended for longitudinal studies, in which changes in HR may help to explain changes in arterial stiffness ${ }^{36)}$.

\section{Conclusion}

The augmentation index, derived from PWA, is widely used to investigate systemic arterial stiffness. The AIx is known to be influenced by age, height, gender, MAP and HR. Accordingly, gender- and agespecific reference values have been devised, and it is recommended that the MAP be entered as a covariate in any statistical analysis. The AIx is also commonly statistically adjusted to a HR of $75 \mathrm{~b} \cdot \mathrm{min}^{-1}$ using a correction factor derived from two small sample studies. This approach may be physiologically and statistically inappropriate. First, there appears to be an important physiological chronic interaction between HR and arterial stiffness. Second, not only was the HR correction factor derived using an inappropriate statistical model, the method of correction assumes that the relationship between HR and arterial stiffness is the same for all populations. A more appropriate practice may be to include the HR as an independent predictor or covariate. This approach is particularly recommended for longitudinal studies, in which changes in HR may help to explain changes in arterial stiffness.

\section{Conflicts of Interest}

None.

\section{References}

1) Weber T, O'Rourke MF, Lassnig E, Porodko M, Ammer M, Rammer M, Eber B: Pulse waveform characteristics predict cardiovascular events and mortality in patients undergoing coronary angiography. J Hypertens, 2010; 28: 797-805

2) Papaioannou TG, Karatzis EN, Karatzi KN, Gialafos EJ, Protogerou AD, Stamatelopoulos KS, Papamichael CM, Lekakis JP, Stefanadis CI: Hour-to-hour and week-toweek variability and reproducibility of wave reflection indices derived by aortic pulse wave analysis: implications for studies with repeated measurements. J Hypertens, 2007; 25: 1678-1686

3) O'Rourke MF, Pauca A, Jiang XJ: Pulse wave analysis. Br J Clin Pharmacol, 2001; 51: 507-522

4) Chen CH, Nevo E, Fetics B, Pak PH, Yin FC, Maughan WL, Kass DA: Estimation of central aortic pressure waveform by mathematical transformation of radial tonometry pressure. Validation of generalized transfer function. Circulation, 1997; 95: 1827-1836

5) Marchais SJ, Guerin AP, Pannier BM, Levy BI, Safar ME, London GM: Wave reflections and cardiac hypertrophy in chronic uremia. Influence of body size. Hypertension, 1993; 22: 876-883

6) Oliver JJ, Webb DJ: Noninvasive assessment of arterial stiffness and risk of atherosclerotic events. Arterioscler Thromb Vasc Biol, 2003; 23: 554-566

7) Wilkinson IB, MacCallum H, Hupperetz PC, van Thoor CJ, Cockcroft JR, Webb DJ: Changes in the derived central pressure waveform and pulse pressure in response to angiotensin II and noradrenaline in man. J Physiol, 2001; 530: $541-550$

8) Janner JH, Godtfredsen NS, Ladelund S, Vestbo J, Prescott E: Aortic augmentation index: reference values in a large unselected population by means of the SphygmoCor device. Am J Hypertens, 2010; 23: 180-185

9) Smulyan H, Marchais SJ, Pannier B, Guerin AP, Safar ME, London GM: Influence of body height on pulsatile arterial hemodynamic data. J Am Coll Cardiol, 1998; 31: 1103-1109

10) Wilkinson IB, Mohammad NH, Tyrrell S, Hall IR, Webb DJ, Paul VE, Levy T, Cockcroft JR: Heart rate dependency of pulse pressure amplification and arterial stiffness. Am J Hypertens, 2002; 15: 24-30

11) Wilkinson IB, MacCallum H, Flint L, Cockcroft JR, Newby DE, Webb DJ: The influence of heart rate on augmentation index and central arterial pressure in humans. J Physiol, 2000; 525 Pt 1: 263-270

12) Stoner L, Lambrick DM, Faulkner J, Young J: Guidelines for the Use of Pulse Wave Analysis in Adults and Children. J Atheroscler Thromb, 2013; 20: 404-406

13) Frimodt-Moller M, Nielsen AH, Kamper AL, Strandgaard S: Reproducibility of pulse-wave analysis and pulse-wave velocity determination in chronic kidney disease. Nephrol 
Dial Transplant, 2008; 23: 594-600

14) Papaioannou TG, Stamatelopoulos KS, Georgiopoulos G, Vlachopoulos C, Georgiou S, Lykka M, Lambrinoudaki I, Papamichael CM, Stefanadis CI: Arterial wave reflections during the menstrual cycle of healthy women: a reproducibility study. Hypertension, 2009; 54: 1021-1027

15) Shahin Y, Barakat H, Barnes R, Chetter I: The Vicorder device compared with SphygmoCor in the assessment of carotid-femoral pulse wave velocity in patients with peripheral arterial disease. Hypertens Res, 2013; 36: 208-212

16) Lee NB, Park CG: Reproducibility of regional pulse wave velocity in healthy subjects. Korean J Intern Med, 2009; 24: $19-23$

17) Pereira T, Maldonado J: Comparative study of two generations of the Complior device for aortic pulse wave velocity measurements. Blood Press Monit, 2010; 15: 316-321

18) Crilly M, Coch C, Bruce M, Clark H, Williams D: Indices of cardiovascular function derived from peripheral pulse wave analysis using radial applanation tonometry: a measurement repeatability study. Vasc Med, 2007; 12: 189-197

19) Paul B, Hewitson CL, Woodman RJ, Mangoni AA: Analysis of short-term reproducibility of arterial vasoreactivity by pulse-wave analysis after pharmacological challenge. Clin Exp Pharmacol Physiol, 2009; 36: 49-54

20) London GM, Blacher J, Pannier B, Guerin AP, Marchais SJ, Safar ME: Arterial wave reflections and survival in end-stage renal failure. Hypertension, 2001; 38: 434-438

21) Russo C, Jin Z, Takei Y, Hasegawa T, Koshaka S, Palmieri V, Elkind MS, Homma S, Sacco RL, Di Tullio MR: Arterial wave reflection and subclinical left ventricular systolic dysfunction. J Hypertens, 2011; 29: 574-582

22) Weber T, Auer J, O'Rourke M F, Kvas E, Lassnig E, Lamm G, Stark N, Rammer M, Eber B: Increased arterial wave reflections predict severe cardiovascular events in patients undergoing percutaneous coronary interventions. Eur Heart J, 2005; 26: 2657-2663

23) Avni B, Frenkel G, Shahar L, Golik A, Sherman D, Dishy $\mathrm{V}$ : Aortic stiffness in normal and hypertensive pregnancy. Blood Press, 2010; 19: 11-15

24) Figueroa A, Sanchez-Gonzalez MA, Perkins-Veazie PM, Arjmandi BH: Effects of watermelon supplementation on aortic blood pressure and wave reflection in individuals with prehypertension: a pilot study. Am J Hypertens, 2011; 24: 40-44

25) Gedikli O, Yilmaz H, Kiris A, Karaman K, Ozturk S, Baykan M, Ucar U, Durmus I, Karahan C, Celik S: Circulating levels of relaxin and its relation to cardiovascular function in patients with hypertension. Blood Press, 2009; 18: 68-73
26) Kamran H, Salciccioli L, Gusenburg J, Kazmi H, Ko EH, Qureshi G, Lazar JM: The effects of passive leg raising on arterial wave reflection in healthy adults. Blood Press Monit, 2009; 14: 202-207

27) Kamran H, Lazar JM, Patel R, Maraj I, Berman H, Salciccioli L: The age-dependent contribution of aortic incident and reflected pressure waves to central blood pressure in african-americans. Int J Hypertens, 2011; 2011: 585703

28) Spence VA, Kennedy G, Belch JJ, Hill A, Khan F: Lowgrade inflammation and arterial wave reflection in patients with chronic fatigue syndrome. Clin Sci (Lond), 2008; 114: 561-566

29) Vlachopoulos C, Kardara D, Anastasakis A, Baou K, Terentes-Printzios D, Tousoulis D, Stefanadis C: Arterial stiffness and wave reflections in marathon runners. Am J Hypertens, 2010; 23: 974-979

30) Martin JS, Beck DT, Gurovich AN, Braith RW: The acute effects of smokeless tobacco on central aortic blood pressure and wave reflection characteristics. Exp Biol Med (Maywood), 2010; 235: 1263-1268

31) Heilman K, Zilmer M, Zilmer K, Lintrop M, Kampus P, Kals J, Tillmann V: Arterial stiffness, carotid artery intimamedia thickness and plasma myeloperoxidase level in children with type 1 diabetes. Diabetes Res Clin Pract, 2009; 84: $168-173$

32) Shim CY, Yang WI, Park S, Kang MK, Ko YG, Choi D, Jang Y, Chung N, Ha JW: Overweight and its association with aortic pressure wave reflection after exercise. Am J Hypertens, 2011; 24: 1136-1142

33) Bodlaj G, Pichler R, Brandstatter W, Hatzl-Griesenhofer M, Maschek W, Biesenbach G, Berg J: Hyperthyroidism affects arterial stiffness, plasma NT-pro-B-type natriuretic peptide levels, and subendocardial perfusion in patients with Graves' disease. Ann Med, 2007; 39: 608-616

34) Lantelme P, Mestre C, Lievre M, Gressard A, Milon $H$ : Heart rate: an important confounder of pulse wave velocity assessment. Hypertension, 2002; 39: 1083-1087

35) Stefanadis C, Dernellis J, Vavuranakis M, Tsiamis E, Vlachopoulos C, Toutouzas K, Diamandopoulos L, Pitsavos C, Toutouzas P: Effects of ventricular pacing-induced tachycardia on aortic mechanics in man. Cardiovasc Res, 1998; 39: 506-514

36) Sa Cunha R, Pannier B, Benetos A, Siche JP, London GM, Mallion JM, Safar ME: Association between high heart rate and high arterial rigidity in normotensive and hypertensive subjects. J Hypertens, 1997; 15: 1423-1430

37) Raudenbush SW, Bryk AS: Hierarchical Linear Models: Applications and Data Analysis Methods (Advanced Quantitative Techniques in the Social Sciences), SAGE Publications, Thousand Oaks, CA, 2001 